\title{
Vibration reduction in lightweight floor/ceiling systems with a sand-sawdust damping layer
}

\author{
Hyuck CHUNG ${ }^{1}$; Grant EMMS ${ }^{2}$ \\ ${ }^{1}$ Auckland University of Technology, New Zealand \\ ${ }^{2}$ Forest Research Institute, New Zealand
}

\begin{abstract}
This paper shows how to use a mathematical model to predict the vibration of lightweight timber-framed floor/ceiling systems (LTFSs) caused by mechanical excitation. The LTFS considered here is made up of an upper floor layer, a cavity space with timber joists and a ceiling. These components are joined by timber battens, ceiling furring channels and ceiling clips. The vibration in the structure is caused by a localized excitation on the top surface and the resulting vibration level of the ceiling surface will be analysed. The cavity space is filled with fibre infill for damping the sound transmitting through the cavity. A unique feature of the design and the model is the sand-sawdust mixture in the upper layer. The theoretical model and the experimental measurements show that the sand-sawdust dampens the vibration in the frequency range between 10 and $200 \mathrm{~Hz}$. The damping by the sand-sawdust and the fibre infill are found by comparing the numerical simulations against the experimental measurements. We show that the simple linear frequency dependent loss factors can be used to predict the low-frequency vibrations of LTFSs.
\end{abstract}

Keywords: Sound, Insulation, Transmission $\quad$ I-INCE Classification of Subjects Number(s): 51.4

\section{INTRODUCTION}

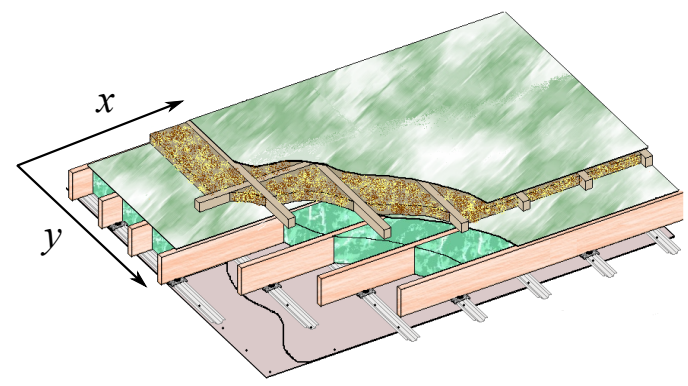

Figure 1 - Depiction of an LTFS. The upper layer has a sand-sawdust mixture enclosed by plywood panels and timber battens. The joists are laminated timber beams. The ceiling panels are attached to the joists by furring channels and resilient rubber clips. The coordinate system later used in mathematical modelling is shown with the $x$ and $y$ axes.

Most residential buildings can be classified as either concrete-based or lightweight timber-framed systems. The concrete-based systems primarily use concrete slabs for walls and floors, and the lightweight systems use timber-framed composite structures. In this paper we study the sound insulation performances of lightweight timber-framed floor/ceiling systems (LTFSs) depicted in Fig. 1. Such LTFSs have become popular due to their ease of construction and environmentally friendly use of timber. As the popularity of the systems grows, their weakness in sound insulation in the low-frequency range has also become apparent. In addition to experimental studies, a theoretical model capable of predicting the performance of many design variations is needed. Such a

\footnotetext{
${ }^{1}$ hchung@aut.ac.nz

${ }^{2}$ grant.emms@scion. co.nz
} 
model requires mechanical and material parameters of all components. Those parameters are usually frequency dependent and may not be measured easily.

According to the listening tests performed on a group of people in (1), the LTFS based on Fig. 1 has better sound insulation than a solid concrete slab of $150 \mathrm{~mm}$ with carpet and a suspended ceiling. The sand-sawdust in the upper layer of the system improves the acoustic performance. In order to put the theoretical model to real use, the mechanical properties of the components, the sand-sawdust layer in particular, need to be quantified. The effects of the sand-sawdust layer is included in the model as a damping coefficient or an imaginary part of the stiffness of the upper layer. We use the experimental data and the theoretical model to show that the damping can be modelled as a linear function of frequency. It is commonly believed that LTFSs cannot perform as well as concrete counterparts. Blazier and DuPree (2) claimed that it was impossible to build a practical LTFS, which could satisfy an average resident. However, the prediction model used in that paper was unsuited for LTFSs.

We use the classical theories of elastic plates and beams to represent the panels, joists and battens (see (3) and (4)). Furthermore the theory of room acoustics is used to include the sound transmitted via the cavity, using the Helmholtz equation. The displacement of individual components and the acoustic pressure are expressed using the Fourier series over the two dimensional rectangular shape of the structure. Our method of using the Fourier series is an extension of the methods in $(5,6,7)$, which deal with ribbed plates with simpler designs. We compute the displacement of the structure (floor and ceiling surfaces) at each frequency. As a result we are able to compute the details of the mode shapes at every frequency, and thus able to compare the theoretical model and the experimental measurements precisely.

The performance of the model is evaluated by its ability to predict particularly the first few resonant frequencies and amplitudes. We have found that our model can predict the first three resonant frequencies within $1.0 \mathrm{~Hz}$ and their amplitudes within $3.0 \mathrm{~dB}$. The resonances of the structure are not distinct after the third resonance. Although the model can predict the decaying rate of the vibration at the frequencies above $80 \mathrm{~Hz}$, it cannot exactly determine the amplitude at a given frequency. We suspect that inhomogeneity and the uncertainties in the structure begin to affect the behaviour. Thus, it may not be possible to completely determine the vibration level at this frequency range. The effects of the sand-sawdust in the upper layer is included in the model as the loss factor, which is the imaginary part of the rigidity of the upper layer. The loss factor here is dependent on the frequency, so that the sand-sawdust dampens the vibration more as the frequency increases. The effects of the fibre-infill in the cavity is similarly included as the imaginary part of the mass density and the speed of sound in the Helmholtz equation.

\section{EXAMPLES OF LTFSS}

The LTFSs in Fig. 2 have the upper layer is modified from a single plywood to a sand-sawdust filled-layer in order to evaluate the damping effects of the sand-sawdust layers. The design of Fig. 2(right) with a sandsawdust layer has given the best sound insulation performances in both physical vibration measurements and subjective listening tests.

During the experimental programme in (1), altogether 26 LTFSs were built and tested. Each of those designs had small variations from the designs shown in Fig. 2. The experimental designs were determined based on the numerical simulations (see (8)) and the building practicality. For example, the simulations prior to the experiments had shown that increasing the cavity depth would have had little effects on the vibration level of the ceiling. Thus the cavity depth was not changed for the designs studied here. The theoretical simulations also showed that the rubber clips and the furring channels on the ceiling reduced the vibration levels of the ceiling considerably, and thus they were always included in the designs. The descriptions of the commercial products used in these LTFSs are given in Appendix.

\section{THEORETICAL MODELLING}

\subsection{Equations for the components}

Our model is made up of the following components given in Table. 1. The $z$-axis points downward so that the upper layer is at $z=0$ and the ceiling is at $z=d$, where $d$ is the depth of the cavity. The top layer is excited by time-harmonic forcing with radial frequency $\omega=2 \pi f$ at $\left(x_{0}, y_{0}\right)$, where $f$ is the frequency in $\mathrm{Hz}$, and thus the whole system is driven at the same frequency $f$. The displacement of a plate for example is then given by $\operatorname{Re}\left[w_{\mathbf{u}}(x, y) e^{\mathrm{j} \omega t}\right]$ for $(x, y) \in[0, A] \times[0, B]$ where $A$ and $B$ are the width and length of the LTFS. Note that the velocity of the top surface is given by $\operatorname{Re}\left[\mathrm{j} \omega w_{\mathbf{u}}(x, y) e^{\mathrm{j} \omega t}\right]$. 

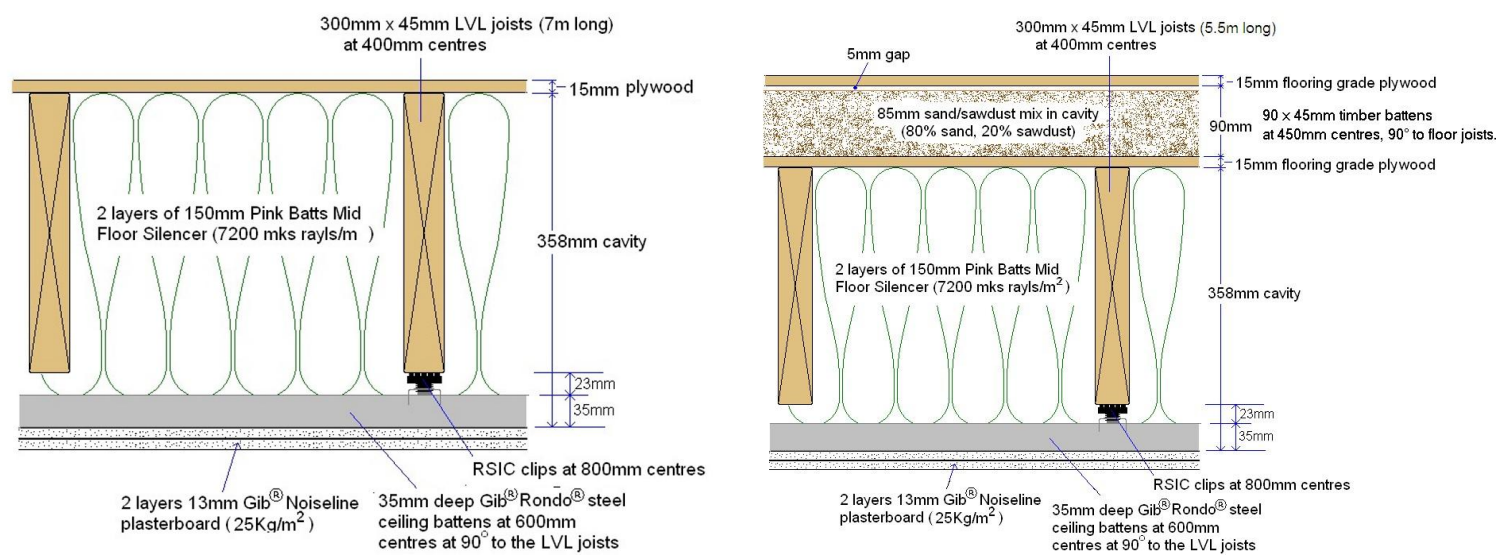

Figure 2 - Cutaway schematics of an LTFS with (left) a single plywood upper layer. The joists span $7.0 \mathrm{~m}$, and the width is $3.2 \mathrm{~m}$ and (right) a sand-sawdust upper layer. The length of the structure is changed to 5.5 meters.

Table 1 - Notations for the elastic plates and beams.

\begin{tabular}{ccccc}
\hline Component & Displacement & Density & Thickness & Modulus \\
\hline Upper layer & $w_{\mathbf{u}}(x, y)$ & $m_{\mathbf{u}}$ & $h_{\mathbf{u}}$ & $E_{\mathbf{u}}$ \\
Timber battens & $w_{\mathbf{t}}\left(x_{i}, y\right)$ & 0 & $h_{\mathbf{t}}$ & $E_{\mathbf{t}}$ \\
Joists & $w_{\mathbf{j}}\left(x, y_{j}\right)$ & $m_{\mathbf{j}}$ & $h_{\mathbf{j}}$ & $E_{\mathbf{j}}$ \\
Furring channels & $w_{\mathbf{f}}\left(x_{i}, y\right)$ & 0 & $h_{\mathbf{f}}$ & $E_{\mathbf{f}}$ \\
Ceiling & $w_{\mathbf{c}}(x, y)$ & $m_{\mathbf{c}}$ & $h_{\mathbf{c}}$ & $E_{\mathbf{c}}$ \\
\hline
\end{tabular}

The displacement of the two plates $w_{\mathbf{u}}$ and $w_{\mathbf{c}}$ satisfies the following thin plate equation (see (4)).

$$
\begin{aligned}
\left(D_{\mathbf{u}} \nabla^{4}-m_{\mathbf{u}} \omega^{2}\right) w_{\mathbf{u}}(x, y) & =F \boldsymbol{\delta}\left(x_{0}, y_{0}\right)-P_{\mathbf{u}}(x, y)-p(x, y, 0) \\
\left(D_{\mathbf{c}} \nabla^{4}-m_{\mathbf{c}} \omega^{2}\right) w_{\mathbf{c}}(x, y) & =P_{\mathbf{c}}(x, y)+p(x, y, d)
\end{aligned}
$$

where $F$ is the external force amplitude, $P_{\mathbf{u}}$ and $p$ denote the force from the attached joists and the acoustic pressure from the cavity, respectively. The localized forcing is expressed by the Dirac delta function $\delta\left(x_{0}, y_{0}\right)=$ $\delta\left(x-x_{0}, y-y_{0}\right)$. We note that ten forcing points are chosen randomly to compute the mean value of $w_{\mathbf{u}}$ in the numerical simulations. The derivation of the above equations can be found in structural acoustics text books such as $(3,9)$. In Eq. (2), $P_{\mathbf{c}}$ and $p(x, y, d)$ denote the force from the battens and the acoustic pressure, respectively. The ceiling is made up of two layers of plasterboard in this case. We again simplify the model by assuming that the ceiling is a single plate with two layers of varying stiffness. The differential operator is defined by $\nabla^{4}=\frac{\partial^{4}}{\partial x^{4}}+2 \frac{\partial^{4}}{\partial x^{2} \partial y^{2}}+\frac{\partial^{4}}{\partial y^{4}}$.

The flexural rigidity, $D_{\mathbf{u}}$, for the upper layer is computed by $E_{\mathbf{u}} h_{\mathbf{u}}^{3} / 12\left(1-v^{2}\right)$, where $E_{\mathbf{u}}, h_{\mathbf{u}}$ and $v$ are the Young's modulus, thickness and the Poisson ratio, respectively. The Young's modulus of the upper layer has to be adjusted because of the additional sand-sawdust layer. Thus we model the upper layer as one plate with stiffening beams (representing battens). This upper layer plate has a bending stiffness equivalent to two plates separated by a gap. Damping by the sand-sawdust is included as the imaginary part of the stiffness, denoted by $\delta_{\mathbf{s}}$. We have the adjusted stiffness $D_{\mathbf{u}}=E\left(h_{\mathbf{u}}^{3}-h_{\mathbf{t}}^{3}\right)\left(1+\mathrm{j} \delta_{\mathbf{s}}\right) / 12\left(1-v^{2}\right)$, where $h_{\mathbf{t}}$ is the thickness of the timber battens. The neutral plane of deformation is assumed to be at the middle of the upper layer. The mass of the timber battens in the upper layer is neglected because they are much lighter than the rest of the upper layer. Thus we have the following equation for the timber battens in the upper layer.

$$
E_{\mathbf{t}} I_{\mathbf{t}} \frac{d^{4}}{d y^{4}} w_{\mathbf{t}}\left(x_{i}, y\right)=P_{\mathbf{t}}(i, y), i=1,2, \ldots, S_{\mathbf{t}}
$$

where $I_{\mathbf{t}}$ is the moment of inertia of the battens. The locations of the timber battens are given by $x_{i}, i=1,2, \ldots, S_{\mathbf{t}}$, where $S_{\mathbf{t}}$ is the number of battens. The force $P_{\mathbf{t}}(i, y)$ comes from the two plywood layers. This is a simplification of the actual design which has the ribbed double-leaf plate configuration. We have made this compromise because the upper layer is thin and light compared to the rest of the structure. 
We set the number of joists $S_{\mathrm{j}}$ and located at $y=y_{j}, j=1,2, \ldots, S_{\mathrm{j}}$. Then the displacement of $j$ th joist, $w_{\mathbf{j}}\left(x, y_{j}\right)$ satisfies the Euler beam equation

$$
\left[E_{\mathbf{j}} \mathrm{I}_{\mathbf{j}} \frac{d^{4}}{d x^{4}}-m_{\mathbf{j}} \omega^{2}\right] w_{\mathbf{j}}\left(x, y_{j}\right)=P_{\mathbf{j}}(x, j)
$$

for $j=1,2, \ldots, S_{\mathbf{j}}$. The moment of inertia $I_{\mathbf{j}}$ is computed by $h_{\mathbf{j}}^{3} d_{\mathrm{j}} / 12$, where $d_{\mathbf{j}}$ is the width of the beams. On the right hand side, $P_{\mathrm{j}}$ denotes the total force applied on the joists from the upper layer ad the ceiling attachments.

The furring channels are light and thin, and thus we assume that they only give additional stiffness to the ceiling. The elasticity of the rubber clips determines the coupling force $P_{\mathrm{j}}$ (see (10)). The equations for the furring channels are

$$
E_{\mathbf{f}} I_{\mathbf{f}} \frac{d^{4}}{d y^{4}} w_{\mathbf{f}}\left(x_{i}, y\right)=P_{\mathbf{f}}(i, y)
$$

for $i=1,2, \ldots, S_{\mathbf{f}}$. The locations of the furring channels (and the rubber clips) are given by $x_{i}, i=1,2, \ldots, S_{\mathbf{f}}$, where $S_{\mathfrak{f}}$ is the number of furring channels. Note that the same notation $x_{i}$ is used for the timber battens in the upper layer, however the indices are distinguished by the notations $S_{\mathbf{t}}$ and $S_{\mathbf{f}}$.

The cavity air acts as primary path of sound at low-frequencies, and thus it must be included in the model. The acoustic pressure, $p(x, y, z)$, in the cavity satisfies the Helmholtz equation (see (11)).

$$
\left(\nabla^{2}+\mu^{2}\right) p(x, y, z)=0
$$

where $\mu=\omega / c$, and $c$ is the speed of sound of the cavity air. The cavity walls are assumed to be acoustically hard, that is the normal derivatives on the walls are zero. Thus we have the following boundary conditions for the normal derivatives of $p$ on the side-walls of the cavity.

$$
p_{x}(0, y, z)=0, p_{x}(A, y, z)=0, p_{y}(x, 0, z)=0, p_{y}(x, B, z)=0 .
$$

We have not considered the effects of sub-diving the cavity by the joists, because the wavelengths at lowfrequency vibration are long compared to the spacing of the joists (600 $\mathrm{mm}$ centres). The coupling conditions between the plates (upper and ceiling) and the air are given by

$$
p_{z}(x, y, z)=\omega^{2} \rho w_{\mathbf{u}}, p_{z}(x, y, d)=\omega^{2} \rho w_{\mathbf{c}},
$$

where $\rho$ is the mass density of the air. The mass density and $\mu$ in the cavity are modified to account for the sound damping by the glassfibre fill.

The equations derived above need to be coupled using the physical conditions at the junctions. Figure 3 shows how the coupling conditions are simplified for the mathematical model. Details of modelling and derivation of equations can be found in (10)

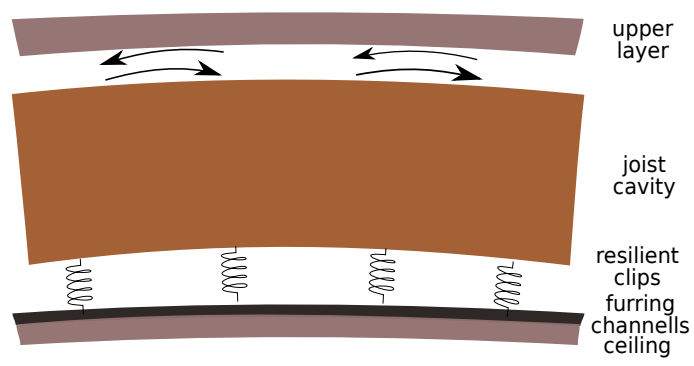

Figure 3 - Depiction of the modelling regime of the junctions between components.

\subsection{Fourier series expansion}

We use the Fourier expansion method to compute the solution of the system of equations given in the previous section. The orthogonal sine-basis functions are given by $\phi_{m}(x)=\sqrt{2 / A} \sin k_{m} x$ and $\psi_{n}(y)=$ $\sqrt{2 / B} \sin \kappa_{n} y$ for $m, n=1,2, \ldots$, , where the wavenumbers are $k_{m}=\pi m / A$ and $\kappa_{n}=\pi n / B$. These modes satisfy the simply-supported conditions. 
The displacement of the upper plate and the ceiling can be expressed by

$$
w_{\mathbf{u}}(x, y)=\sum_{m, n=1}^{N} c_{m n}^{\mathbf{u}} \phi_{m}(x) \psi_{n}(y), w_{\mathbf{c}}(x, y)=\sum_{m, n=1}^{N} c_{m n}^{\mathbf{c}} \phi_{m}(x) \psi_{n}(y),
$$

where $\left\{c_{m n}^{\mathbf{u}}\right\}$ and $\left\{c_{m n}^{\mathbf{c}}\right\}$ are complex valued coefficients and $N$ is the truncated number of modes we use to compute the solutions. Substituting Eq. (9) to the equations of the individual components and using the orthogonality will lead to a system of algebraic equations for $\left\{c_{m n}^{\mathbf{u}}, c_{m n}^{\mathbf{c}}\right\}$, in other words we will have an equation for the vectors, $\boldsymbol{c}_{\mathbf{u}}$ and $\boldsymbol{c}_{\mathbf{c}}$ of $\left\{c_{m n}^{\mathbf{u}}\right\}$ and $\left\{c_{m n}^{\mathbf{c}}\right\}$, respectively. We have assumed that the upper layer and the joists are always in contact. Then we have $w_{\mathbf{u}}\left(x, y_{j}\right)=w_{\mathbf{j}}(x, j)$. Similarly the furring channels and the ceiling are assumed be always in contact, and thus we have $w_{\mathbf{c}}\left(x_{i}, y\right)=w_{\mathbf{f}}(i, y)$. We then need to find the coefficients $\left\{c_{m n}^{\mathbf{u}}, c_{m n}^{\mathbf{c}}\right\}$ to find the displacement everywhere in the structure. Note that the series solutions are truncated to $N$ terms for computation. It was found that $N=20$ to be sufficient for the frequency range studied here.

The acoustic pressure in the cavity space is also expressed using the Fourier expansion, which uses cosine basis function, $\alpha_{m}(x)=\sqrt{2 / A} \cos k_{m} x$ and $\beta_{n}(x)=\sqrt{2 / B} \cos \kappa_{n} y$ for $m, n=0,1, \ldots$ These modes satisfy the acoustically-hard wall conditions at the cavity walls. The acoustics pressure is

$$
p(x, y, z)=\sum_{m, n=0}^{N}\left\{\Gamma_{m n}^{(1)} e^{\gamma_{m n} z}+\Gamma_{m n}^{(2)} e^{-\gamma_{m n} z}\right\} \alpha_{m}(x) \beta_{n}(y)
$$

where $\left\{\Gamma_{m n}^{(1)}, \Gamma_{m n}^{(2)}\right\}$ are the coefficients to be determined and the wavenumbers are defined by $\gamma_{m n}=\sqrt{k_{m}^{2}+\kappa_{n}^{2}-k^{2}}$. Finally, substituting the expansions given by Eqs. (9) and (10) into the partial differential equations derived in the previous section will give us a system of linear equations for $\left\{c_{m n}^{\mathbf{u}}, c_{m n}^{\mathbf{c}}, \Gamma_{m n}^{(1)}, \Gamma_{m n}^{(2)}\right\}$. The matrices corresponding to the above formulations are given Appendix of (10).

The root-mean-square velocity of the ceiling surface, which will be compared against the experimental results, is computed by the integration of the square of the velocity given by Eq. 11. The integral was computed numerically once the values of $v(x, y)=\mathrm{j} \omega w_{\mathbf{c}}(x, y)$ over the ceiling surface had been computed.

$$
\sqrt{\left\langle|v|^{2}\right\rangle}=\sqrt{\int_{0}^{B} \int_{0}^{A}|v(x, y)|^{2} d x d y}
$$

\section{DETERMINING THE PARAMETERS FOR THE CAVITY, CEILING ATTACHMENTS AND THE SAND-SAWDUST LAYER}

\subsection{Experimental set-up}

On each floor an electrodynamic shaker was used to provide a vertical force on the upper floor surface. The shaker was connected to the floor through a wire stinger and a reference force transducer. The stinger is there to ensure that only vertical forces are transmitted in the floor, while the force transducer lets us know how much force is sent into the floor. The shaker body was mounted on a beam which straddled the floor and rested on supports which sat on the concrete collar surrounding the floor. Vibration isolation of the beam from the concrete collar was provided by very resilient pads made of polyester fibre infill. The shaker was driven with pseudo-random signal with a bandwidth from $10 \mathrm{~Hz}$ to $500 \mathrm{~Hz}$, for a duration of 2 seconds (to achieve a frequency resolution of $0.5 \mathrm{~Hz}$ ). The position on the floor was selected so that the low-frequency modes would be excited. Only one position on each floor was chosen. It is often useful to select two or more positions on a structure to ensure a sufficient number of modes are excited, and to act as a check for results. However, in this case, it would have taken too long to do two complete vibration response scans of each floor.

A scanning laser vibrometer (Polytec PSV 300) was used to measure the velocity of the floor upper surface vibration and the ceiling vibration in a direction which is normal to the surfaces. A grid with a spatial resolution of 10-14 cm was used to obtain a map of the surface velocity of the floor and ceiling relative to the input force; both amplitude and phase information was recorded at each frequency. The laser vibrometer measurement equipment was connected to the force transducer so that the recorded surface vibration is normalised with respect to the force applied. Therefore the results shown in the following sections are the transfer function between the velocity and the input force. The signal sent to the shaker was matched with the sampling time of the laser vibrometer software. This ensured minimal spectral leakage and a frequency resolution of $0.5 \mathrm{~Hz}$. 


\subsection{Cavity fibre infill}

The effect of the fibrous infill in the cavity is modelled using a complex propagation constant $\mu$ in Eq. (6). This complex propagation constant can be determined from existing models of sound propagation in porous media. An overview and application of such models is given in (12) and (13). Most porous media models assume that the porous media forms a rigid frame, within which the air moves. This rigid-frame assumption is valid for high frequencies. In our case, however, we consider low frequencies, where the wavelength may be larger than the porous media thickness, and thus we cannot necessarily assume that the porous media is not in motion. The Biot theory describing wave motion in an elastic porous media can be used in this more complex case, where the frame is not assumed to be rigid (see (12)). However, for the purposes of this paper and our model, we did not go to the complexity of using the Biot theory, but based our cavity model on the empirical model and results presented in Appendix C of (13).

When the wavelength of sound in the fibrous infill is less than the thickness of the infill we can assume that the fibrous infill is rigid and unmoving, and $\mu$ in the cavity is modified to

$$
\mu=\frac{2 \pi f}{c_{0}} \sqrt{\frac{1-(1-\gamma) \eta}{1+\sigma}}
$$

where $c_{0}$ is the speed of sound in air, $\gamma$ is the ratio of specific heats for the gas ( $=1.4$ for air), and

$$
\eta=0.592 a\left(X_{1}\right)+\mathrm{j} b\left(X_{1}\right), \sigma=a(X)+\mathrm{j} b(X)
$$

where $X=\rho_{0} f / R_{1}$ and $X_{1}=0.856 \rho_{0} f / R_{1} . R_{1}$ is the flow resistivity, and $\rho_{0}$ is the air density. The functions $a(X)$ and $b(X)$ in Eq.(13) are defined by

$$
a(X)=\frac{T_{3}\left(T_{1}-T_{3}\right) T_{2}^{2}-T_{4}^{2} T_{1}^{2}}{T_{3}^{2} T_{2}^{2}+T_{4}^{2} T_{1}^{2}}, b(X)=\frac{T_{1}^{2} T_{2} T_{4}}{T_{3}^{2} T_{2}^{2}+T_{4}^{2} T_{1}^{2}},
$$

where $T_{1}=1+9.66 X, T_{2}=X+0.0966 X^{2}, T_{3}=2.537+9.66 X, T_{4}=0.1591(1+0.7024 X)$.

When the wavelength of sound in the fibrous infill is greater than the thickness of the infill, the fibrous infill can no longer be regarded as rigid and unmoving. As a consequence the absorption is reduced compared to the short wavelength case. Appendix $\mathrm{C}$ of (13) gives results of expected transmission loss for porous layers in the low-frequency range when the porous material can no longer be regarded as rigid. These results suggest that the transmission loss (in $\mathrm{dB}$ ) tends to zero as the frequency tends to zero. Thus there is a smooth transition to the high-frequency range for transmission loss against the log of the frequency. We also note that the flow resistivity of a porous medium is related to the pressure drop across the porous medium, which does not only apply to static flow but also to oscillations at low frequencies (see (9)). Therefore, at low frequencies the transmission loss across the porous material $\Delta L_{p}$ is approximately related to the flow resistivity $R_{1}$ by $10^{\Delta L_{p} / 20} \propto R_{1}$

We may define a modified flow resistivity $\widetilde{R}_{1}$ for low-frequencies. $\widetilde{R}_{1}$ is inserted into Eq. (12) to produce $\mu$. We use the results in Appendix $C$ of (13) for the transmission loss in porous materials at low frequencies and assume that $\widetilde{R}_{1}$ is zero at $f=0$ and linearly increasing until the wavelength of sound in the cavity $\lambda_{m}$ is equal to the thickness of the fibrous infill in the cavity $d$ at which point $\widetilde{R}_{1}=R_{1}$ :

$$
\widetilde{R}_{1}=\left\{\begin{array}{ccc}
R_{1} f / f_{K} & \text { for } \quad f<f_{K} \\
R_{1} & \text { for } \quad f \geq f_{K}
\end{array}\right.
$$

where $f_{K}$ is the frequency at which $\lambda_{m}=d$ and is numerically determined using Eq. (12). For the flow resistivity of 7200 Rayls/m and thickness of $300 \mathrm{~mm}$, we have $f_{K}=980 \mathrm{~Hz}$.

The floor shown in Fig. 2(left) has a ceiling consisting of two layers of $13 \mathrm{~mm}$ plasterboard screwed to furring channels at $600 \mathrm{~mm}$ centres, which are attached to every other joist (i.e. at $800 \mathrm{~mm}$ centres) through resilient clips. Our model assumes that there is a ceiling clip on every joist, which is compensated for by reducing the stiffness of each clip by a suitable amount. The cavity is $358 \mathrm{~mm}$ deep and is filled with two layers of $150 \mathrm{~mm}$ sound control type fibreglass. The experimental test version of this floor had ceiling furring channels $35 \mathrm{~mm}$ deep and a calculated stiffness of $11000 \mathrm{Nm}^{2}$. The ceiling furring resilient clips have a measured stiffness of $220000 \mathrm{Nm}^{-1}$ with a $130 \mathrm{~N}$ constant load, and a loss factor of about 0.1 . The cavity infill has a flow resistivity of 7227 Raylm$^{-1}$ and a density of $12 \mathrm{kgm}^{-3}$. Figures $4(\mathrm{a}, \mathrm{b})$ show the difference between the constant and linear modelling of the flow resistivity. Setting the flow resistivity constant gives poor agreement at low-frequencies with the experiment as shown in Fig. 4(a). Figure 4(b) shows that the linear model gives better agreement at the low-frequencies, though the behaviour at higher frequencies changes little. The theoretical modelling results are obtained by simulating ten random excitation locations on the top layer. 
(a)

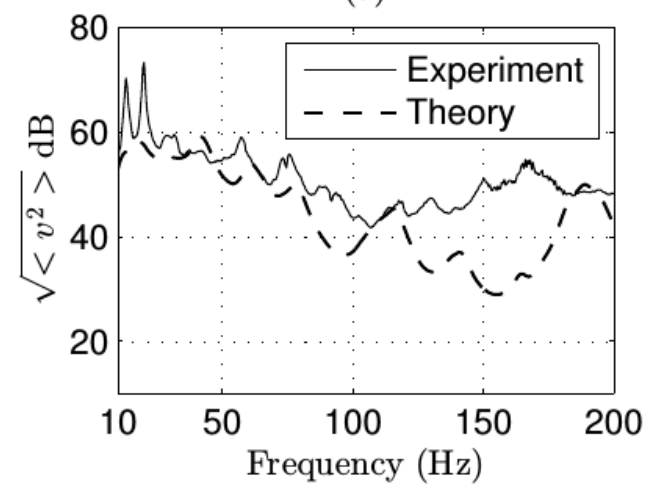

(b)

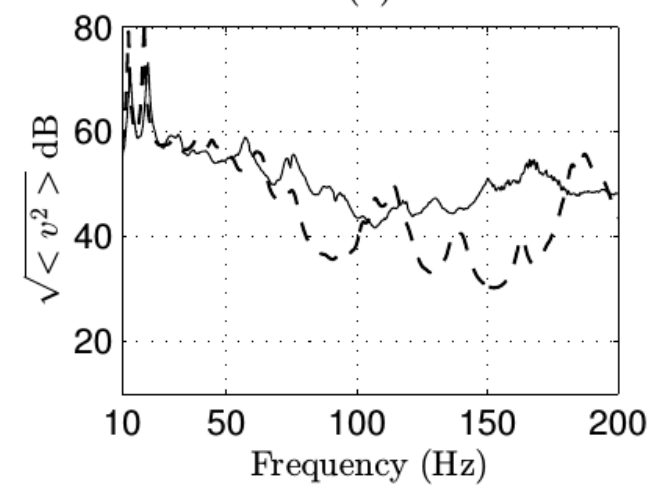

Figure 4 - The root-mean-square velocity of the ceiling of the system shown in Fig. 2. (a) the flow resistivity is constant 7200 Rayls $\mathrm{m}^{-1}$, (b) the flow resistivity is modelled by Eq. (14). The theoretical modelling results are shown by the dashed line, and the measurements are shown by the solid line.

\subsection{Sand-sawdust layer}

Figure 2(right)) shows a floor with $90 \mathrm{~mm}$ deep battens separate two layers of $15 \mathrm{~mm}$ plywood, and in-filled between the battens is an $85 \mathrm{~mm}$ layer of paving sand combined with sawdust with an 80/20 mix ratio by volume. Mixing sawdust in with the sand gives better damping than using sand alone and helps prevent the sand compacting. The model is not able to represent the upper surface exactly as built, but is able to model an equivalent single upper surface plate with rib stiffeners. We estimated the loss factor of the upper surface of the floor for frequencies above $100 \mathrm{~Hz}$ by measuring the amplitudes of the surface vibrations of the floor and the rate of decay as the vibrations propagated from the shaker excitation point. We averaged the decay measurements performed in directions parallel and perpendicular to the joist directions to produce a single value of the loss factor estimate. For frequencies $100 \mathrm{~Hz}$ and above we therefore determined the loss factor to be between 0.4 and 0.8 . This seems to be a large value for loss factor, but such values are reported by Richards and Lenzi (14) for pure sand on plates, and by Yanagida et al. (15) for binary powder mixtures (including sand and rubber powder). At the fundamental bending frequency of the system we determined the loss factor to be 0.1 by measuring the width of the fundamental peak.

Sun et al. (16) and Lin et al. (17) showed that the damping due to the sand layer on a plate is frequency dependent. Thus we expect the same for the mixture and try several functions of frequency to model the damping.

$$
\begin{aligned}
\delta_{\mathrm{s}} & =0.4, \\
\delta_{\mathrm{s}} & =\frac{0.8}{200} f, \\
\delta_{\mathrm{s}} & =\frac{0.4}{200} f .
\end{aligned}
$$

These functions are simplifications of a not very well understood phenomenon. Figure 5 show the comparison between the models with the constant damping and the frequency dependent damping given by Eqs. (15) and (17), respectively. The modelling results in Fig. 5 shows that the frequency dependent damping for the sand-sawdust layer gives better agreement with the measurements, around the first two resonant peaks (between 10 and $30 \mathrm{~Hz}$ ). There are, however, significant deviations from the measurement results for some of the resonances between 30 and $50 \mathrm{~Hz}$. This is probably due to inaccuracies of the estimated loss factor of the sand and sawdust fill.

\section{DISCUSSION: ADVANTAGES OF USING SAND-SAWDUST}

We emphasize the effectiveness of the sand-sawdust layer in damping vibration in Fig. 6 by comparing the performance of a system with a layer of floating gypsum concrete as the upper layer. The gypsum concrete upper layer system has comparable mass $\left(81 \mathrm{kgm}^{-2}\right)$ to the sand-sawdust upper layer system (mass 113 $\mathrm{kgm}^{-2}$ ), but rather less bending stiffness $\left(3.4 \times 10^{4} \mathrm{Nm}\right.$ cf. $\left.1.2 \times 10^{5} \mathrm{Nm}\right)$. Fig. 6 shows the comparison between the gypsum concrete and sand-sawdust systems when the upper layer of the sand-sawdust system is thinner ( $45 \mathrm{~mm}$ gap between plywood layers, and $40 \mathrm{~mm}$ of sand-sawdust mix in the gap). The weight of the upper layer of this thinner sand-sawdust system is $56 \mathrm{kgm}^{-2}$. The comparison in Fig. 6 shows that the damping 

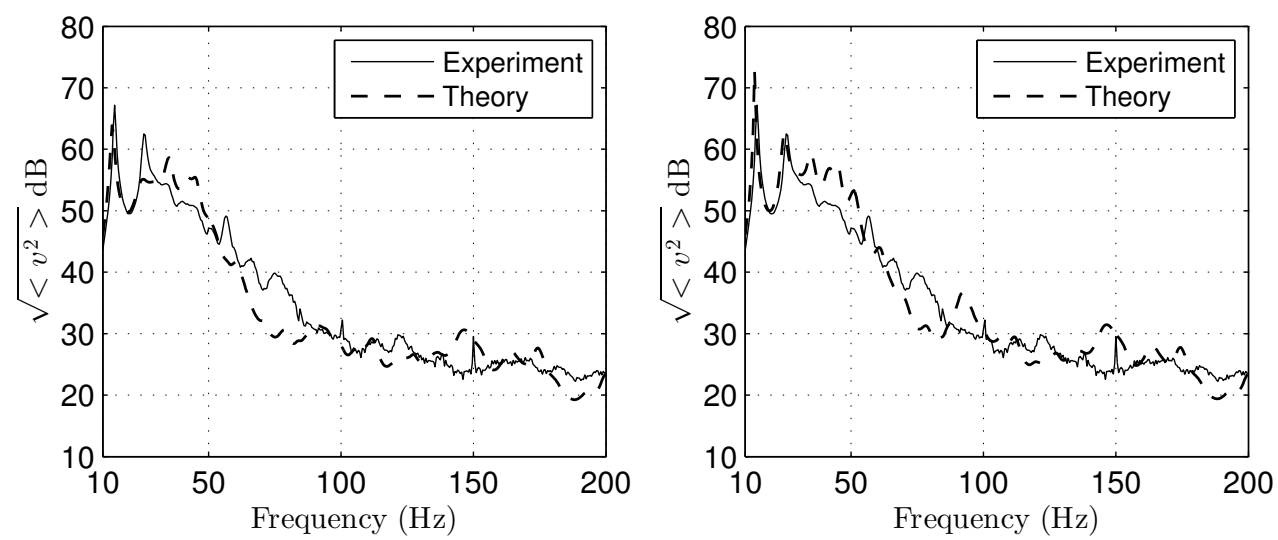

Figure 5 - Comparison of the theoretical (dashed) and measured (solid) root-mean-square velocity of the ceiling when $\delta$ is given by Eq. (15) (left) and Eq. (17) (right).

contribution by the sand-sawdust cannot be replicated by simply adding equivalent mass and stiffness. The sand-sawdust layer dampens the vibration above $60 \mathrm{~Hz}$ more effectively than the concrete upper layer when the weight of the upper layer is similar. One should also note that we obtained good results with the sand-sawdust upper floor system when compared to the gypsum concrete even though the gypsum concrete is a floating raft (on a resilient layer), and the sand-sawdust floor is not - the top plywood layer is screw-fixed to the bottom plywood layer via battens.

Although this paper is about low-frequency vibrations, standard ISO tapping-machine impact sound insulation tests were conducted during the experimental programme in (1). We found that the concrete and sand-sawdust systems had the same $L_{n, w}$ value of $52 \mathrm{~dB}$ when they had similar upper layer stiffness, whereas the thicker (similar weight) sand-sawdust system had an $L_{n, w}$ value of $48 \mathrm{~dB}$.
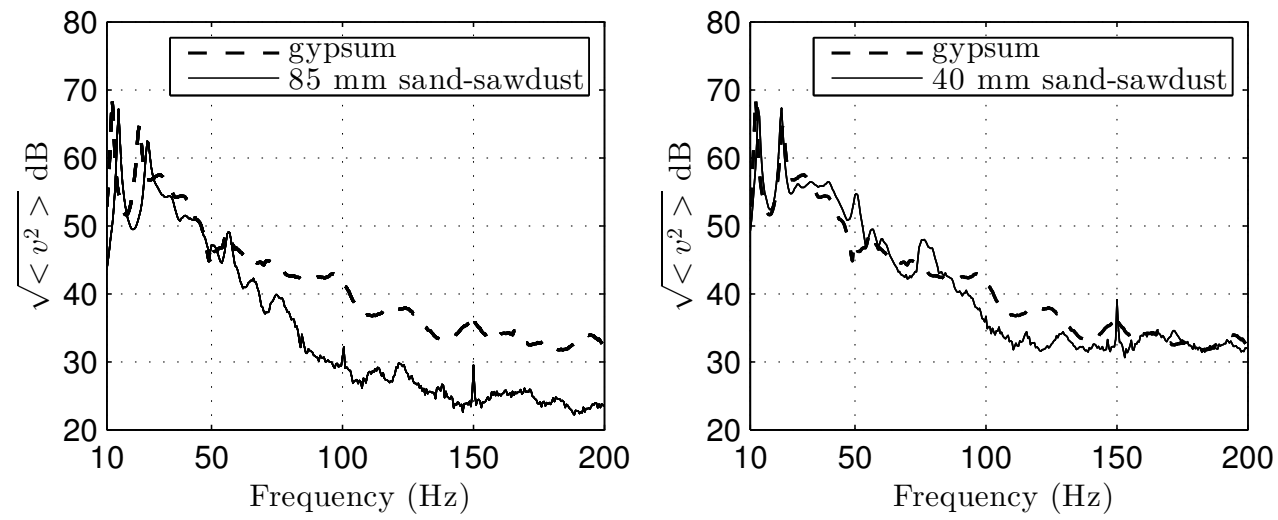

Figure 6 - Comparison of the experimental measurements of the root-mean-square velocity of the ceilings of the LTFSs with sand-sawdust ( $85 \mathrm{~mm}$ thick) and a floating gypsum concrete upper layer with equivalent mass and significantly less bending stiffness (left). The same with sand-sawdust ( $40 \mathrm{~mm}$ thick) and a floating gypsum concrete upper layer with equivalent bending stiffness and significantly greater mass (right).

Figure 7 shows numerical simulations of various stiffness and mass densities of the upper layer. The mass density and the stiffness are varied in order to confirm that the damping by the sand-sawdust cannot be achieved by replacing it with simple mass. Both simulations in Fig. 7 shows that the increase in mass and stiffness certainly lowers the vibration level above $80 \mathrm{~Hz}$. Furthermore, it takes an impractical amount of mass and stiffness to achieve performance comparable to that of sand-sawdust. When observing the vibrations of the floor upper surface, flexural standing waves could be seen in the gypsum concrete topping, whereas the flexural waves in the sand-sawdust floor upper did not propagate far enough to form standing waves (1). Measurements by others on gypsum board show the loss factor to be approximately 0.02 (see (18)). 

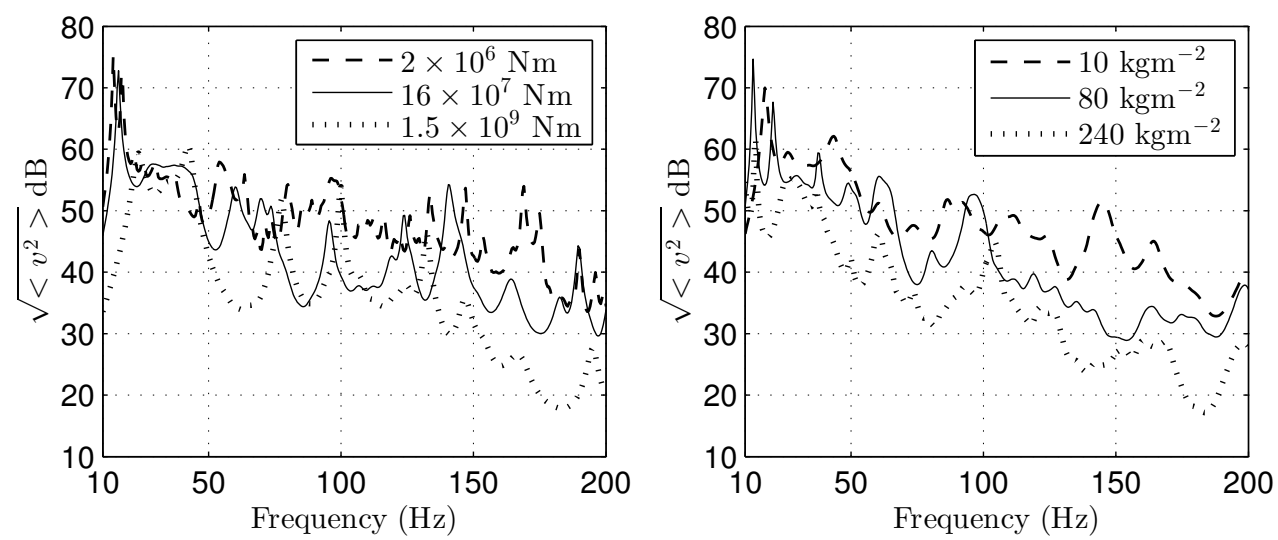

Figure 7 - Numerical simulations of the root-mean-square velocity of the ceiling with various upper layer bending stiffness (left) and mass densities (right).

\section{SUMMARY}

This paper has demonstrated the potential of the vibration damping ability of the sand-sawdust layer in LTFSs. The sand-sawdust layer achieved greater performance than an equivalently massive concrete layer. Thus the benefit of using the sand-sawdust in LTFSs has been confirmed. In order to quantify the damping, a mathematical model is used to determine the loss factor of the sand-sawdust layer. It has been found that using a loss factor as a linear function of frequency gives us a good agreement between theoretical predictions and experimental measurements. In addition, a modelling regime for the fibre-infill in the cavity space has been studied. The flow-resistivity of the infill as a linear function of frequency has given us a good agreement between the theory and the experiments. Although the theories of fibrous media and granular media tell us that there is a complex interaction between the damping and the frequency, simple linear functions have been chosen so that the mathematical model is kept simple and practical.

\section{A. MATERIAL PARAMETERS}

- 15 mm 5-ply Ecoply F11 plywood: Manufacturer's nominal Density $=560 \mathrm{kgm}^{-3}$, nominal static bending stiffness $2360 \mathrm{Nm}^{2}$ along face grain, $684 \mathrm{Nm}^{2}$ perpendicular to face grain assuming 10.5 $\mathrm{GPa}$ along-grain wood stiffness. Dynamic measurements from one sample showed that along-grain wood stiffness was $13 \mathrm{GPa}$. Apparent measured dynamic bending stiffness along face grain (from floor measurements) is equivalent to homogeneous material with Young's modulus from 12 to $14 \mathrm{GPa}$. Vibration loss factor of material assumed to be 0.03 .

- $13 \mathrm{~mm}$ GIB Noiseline (gypsum) plasterboard: Manufacturer's nominal density $=962 \mathrm{kgm}^{-3}$. Dynamic Young's modulus $=3.7 \mathrm{GPa}$. Measured vibration loss factor $=0.013$. Supplied by Winstone Wallboards Ltd.

- Gypsum top layer: USG Levelrock 3500 PS, pre-sanded gypsum concrete. Manufacturers nominal density $=1920 \mathrm{kgm}^{-3}$. Nominal Young's modulus $=6.6 \mathrm{GPa}$.

- Carter-Holt-Harvey (CHH) Hyspan LVL (laminated veneer lumber): Manufacturer's nominal density = $620 \mathrm{kgm}^{-3}$, nominal static Young's modulus = 13.2 GPa. Apparent dynamic Young's modulus from measurements $=14.5 \mathrm{GPa}$ to $15.5 \mathrm{GPa}$. Assumed vibration loss factor $=0.03$.

- $300 \mathrm{~mm}$ CHH Hybeam I-beam (HJ300-63): Manufacturer's nominal linear density $=4.4 \mathrm{kgm}^{-1}$, nominal static bending stiffness $=1111000 \mathrm{Nm}^{2}$. Assumed vibration loss factor $=0.03$.

- $150 \mathrm{~mm}$ Tasman Insulation Mid-floor Silencer: Measured sample flow resistivity $=7227$ Rayls m$^{-1}$. Density $=12 \mathrm{kgm}^{-3}$.

- Sand/sawdust mix (80/20): Density $=1210 \mathrm{kgm}^{-3}$.

- RSIC clip: Dynamic Stiffness at $20 \mathrm{~Hz}$ under $130 \mathrm{~N}$ load (approx equiv to $25 \mathrm{kgm}^{-2}$ ceiling surface density) $=220000 \mathrm{Nm}^{-1}$. Loss factor $=0.1$.

- Gib Rondo Batten: Estimated (from measurements) bending stiffness when attached to plasterboard = $11000 \mathrm{Nm}^{2}$

\section{ACKNOWLEDGEMENT}

The authors thank G. Schmid of Acoustics Research Centre at the University of Auckland for Figs. 1 and 2. 


\section{REFERENCES}

1. Emms G, Chung H, Dodd G, Schmid G, McGunnigle K. FWPRDC Project PN04.2005 Maximising impact sound resistance of timber framed floor/ceiling systems. The Forest and Wood Products Research and Development Corporation. Australian Government; 2006. PN04.2005.

2. Blazier WE, DuPree RB. Investigation of low-frequency footfall noise in wood-frame, multifamily building construction. Journal of the Acoustical Society of America. 1994;96:1521-1532.

3. Cremer L, Heckl M, Petersson BAT. Structure-borne sound: Structural vibrations and sound radiation at audio frequencies. 3rd ed. Berlin: Springer; 2005.

4. Shames IH, Dym CL. Energy and finite element methods in structural mechanics. SI units ed. New York: Taylor \& Francis; 1991.

5. Brunskog J. Acoustic Excitation and Transmission of Lightweight Structures. Lund University; 2002.

6. Mace BR. Periodically stiffened fluid loaded plates, II: Response to line and point forces. Journal of Sound and Vibration. 1980;73(4):487-504.

7. Mace BR. Sound radiation from a plate reinforced by two sets of parallel stiffeners. Journal of Sound and Vibration. 1980;71(3):435-441.

8. Chung H, Emms G. Fourier series solutions to the vibration of rectangular lightweight floor/ceiling structures. Acta Acustica United With Acustica. 2008;94(3):401-409.

9. Fahy F. Sound and structural vibration: Radiation, transmission, and response. London: Academic Press; 1985.

10. Chung H, Emms G, Fox C. Vibration Reduction in Lightweight Floor/Ceiling Systems with a SandSawdust Damping Layer. Acta Acustica united with Acustica. 2014;100(4):628-639.

11. Morse PM, Ingard KU. Theoretical Acoustics. New Jersey, USA: Princeton University Press; 1987.

12. Allard J, Atalla N. Propagation of Sound in Porous Media: Modelling Sound Absorbing Materials 2nd ed. Wiley; 2009.

13. Bies D, Hansen C. Engineering noise control: theory and practice. 4th ed. Taylor \& Francis; 2009.

14. Richards E, Lenzi A. On the prediction of impact noise, VII: The structural damping of machinery. Journal of Sound and Vibration. 1984;97(4):549-586.

15. Yanagida T, Matchett A, Coulthard J. Damping and elastic properties of binary powder mixtures. Powder technology. 2002;127(2):107-115.

16. Sun JC, Sun HB, Chow LC, Richards EJ. Predictions of total loss factors of structures, Part II: loss factors of sand-filled structures. Journal of Sound and Vibration. 1986;104:243-257.

17. Lin ML, Huang TH, You JC. The effects of frequency on damping properties of sand. Soil dynamics and earthquake engineering. 1996;15(4):269-278.

18. Davy J. The measurement of the damping loss factor of gypsum plasterboard. In: Proceedings of the Tenth Asia-Pacific Vibration Conference, 12 - 14 November, Gold Cost Australia, 2003;. . 